УДК 517.524

\title{
The Solution of Algebraic Equations of Continuous Fractions of Nikiports
}

\author{
Vladimir I. Shmoylov* \\ Southern Scientific Center of RAS \\ Chehova, 41, Rostov-on-Don, 344006 \\ Russia \\ Gennadiy A. Kirichenko ${ }^{\dagger}$ \\ Southern Federal University, ITA \\ Nekrasovsky, 44, Taganrog, Rostov region, 347928
}

Russia

Received 06.04.2014, received in revised form 06.07.2014, accepted 06.08.2014

Analytical expressions representing all the roots of a random algebraic equation of $n$-th degree in terms of the equation coefficients are presented in the paper. These formulas consist of two ratios of infinite Toeplitz determinants. The diagonal elements of the determinants are the coefficients of algebraic equations. To find complex roots the method of summation of divergent continued fractions is used.

Keywords: algebraic equation, infinite Toeplitz determinant, $r / \varphi$-algorithm, diverging continuous fractions.

\section{Introduction}

To design modern complex objects it is necessary to analyze their supposed characteristics even in the early stages of their development. Differential and integral equations as well as the classical algebraic equations are used for modeling. Algebraic equation is one of the oldest objects of research in mathematics.

There are various applications of algebraic equations to scientific and technical problems. For example, algebraic equations arise is of interest in studies of equilibrium states of complex thermodynamic and mechanical systems. Algebraic equations are often used in aerodynamics. For example, the rate of climb of a plane is determined by the algebraic equation of eighth degree. Algebraic equations are used in the calculation of the flow over the wing in the Prandtl theory. The degree of the equations depends on the law of variation of the lift coefficient from the angle of attack. The problem of structural stability involves the calculation of eigenvalues of matrices. The eigenvalues are determined from the solution of algebraic equation. The equation degree is equal to the number of harmonics. Algebraic equations most often arise in various geometrical calculations, for example, in determination of the intersection points of two curves, in the design of wings, fuselages, etc. The recently published monograph $[1,2]$ dedicated to the various aspects of the theory and practice of algebraic equations. However, internationally known expert R. Hamming gave, in the book published half a century ago [3], the following remark: "the problem of finding the roots of polynomials occurs frequently enough to warrant a thorough examination and development of special methods for its solution. A whole book can be dedicated to the different known methods of finding a valid linear and quadratic multiplier. The large verity of methods shows that no one of them is completely satisfactory". In fact, there

\footnotetext{
*shmoylov40@atinfotectt.ru

†vtgak@mail.ru

(c) Siberian Federal University. All rights reserved
} 
are more than hundred of algorithms and their modifications that are used for finding zeros of polynomials [4]. Mainly they are algorithms of numerical solution of algebraic equations. The so-called Mellin integral formula [5] is the best known analytical algorithm for solving algebraic equations. Recently the further development of the Mellin integral formula was proposed [6].

The paper presents analytical expressions that represent all the roots of a random algebraic equation of $n$-th degree in terms of the coefficients of the equation. These formulas consist of two ratios of infinite Toeplitz determinants. The diagonal elements of the determinants are the coefficients of algebraic equations. To find complex roots an advanced method of summation of divergent continued fractions, called $r / \varphi$-algorithm, is used [7]. The algorithm is finding application in various areas of computational mathematics [8-11].

\section{Problem statement}

There is an algebraic equation of $n$-th degree:

$$
x^{n}+\alpha_{1} x^{n-1}+\cdots+\alpha_{n-1} x+\alpha_{n}=0 .
$$

Let us write the following generating function:

$$
\frac{1}{1+\alpha_{1} x+\alpha_{2} x^{2}+\cdots+\alpha_{n} x^{n}}=1+c_{1} x+c_{2} x^{2}+\cdots+c_{m} x^{m}+\ldots
$$

Coefficients $\alpha_{i}$ in (1.1) and (1.2) are the same. Coefficients $c_{m}$ of sequence (1.2) can be obtained from the following linear recurrence relations

$$
c_{m}=-\left(\alpha_{1} c_{m-1}+\alpha_{2} c_{m-2}+\cdots+\alpha_{n} c_{m-n}\right), \quad c_{0}=1, c_{1}=-\alpha_{1} .
$$

To determine the roots of algebraic equation (1.1) Aitken proposed the following formula [13]:

$$
\begin{aligned}
& \lim _{m \rightarrow \infty} \frac{c_{m+1}}{c_{m}}=x_{1}, \\
& \lim _{m \rightarrow \infty}\left(\frac{\left|\begin{array}{cc}
c_{m+1} & c_{m+2} \\
c_{m+2} & c_{m+3}
\end{array}\right|}{\left|\begin{array}{cc}
c_{m} & c_{m+1} \\
c_{m+1} & c_{m+2}
\end{array}\right|}: \frac{c_{m+1}}{c_{m}}\right)=\frac{x_{1} x_{2}}{x_{1}}=x_{2}, \\
& \lim _{m \rightarrow \infty}\left(\frac{\left|\begin{array}{lll}
c_{m+1} & c_{m+2} & c_{m+3} \\
c_{m+2} & c_{m+3} & c_{m+4} \\
c_{m+3} & c_{m+4} & c_{m+5}
\end{array}\right|}{\left|\begin{array}{ccc}
c_{m} & c_{m+1} & c_{m+2} \\
c_{m+1} & c_{m+2} & c_{m+3} \\
c_{m+2} & c_{m+3} & c_{m+4}
\end{array}\right|}: \frac{\left|\begin{array}{cc}
c_{m+1} & c_{m+2} \\
c_{m+2} & c_{m+3}
\end{array}\right|}{\left|\begin{array}{cc}
c_{m} & c_{m+1} \\
c_{m+1} & c_{m+2}
\end{array}\right|}\right)=\frac{x_{1} x_{2} x_{3}}{x_{1} x_{2}}=x_{3} .
\end{aligned}
$$

To find $x_{i}$ the following relation is used:

$$
x_{i}=\lim _{m \rightarrow \infty}\left(\frac{H_{i}^{(m+1)}}{H_{i}^{(m)}}: \frac{H_{i-1}^{(m+1)}}{H_{i-1}^{(m)}}\right),
$$

where

$$
H_{i}^{(m)}=\left|\begin{array}{cccc}
c_{m} & c_{m+1} & \ldots & c_{m+i-1} \\
c_{m+1} & c_{m+2} & \ldots & c_{m+i} \\
\ldots & \ldots & \ldots & \ldots \\
c_{m+i-1} & c_{m+i} & \ldots & c_{m+2 i-2}
\end{array}\right|, \quad H_{0}^{(m)}=1 .
$$


Thus, the root $x_{i}$ can be represented by the expression:

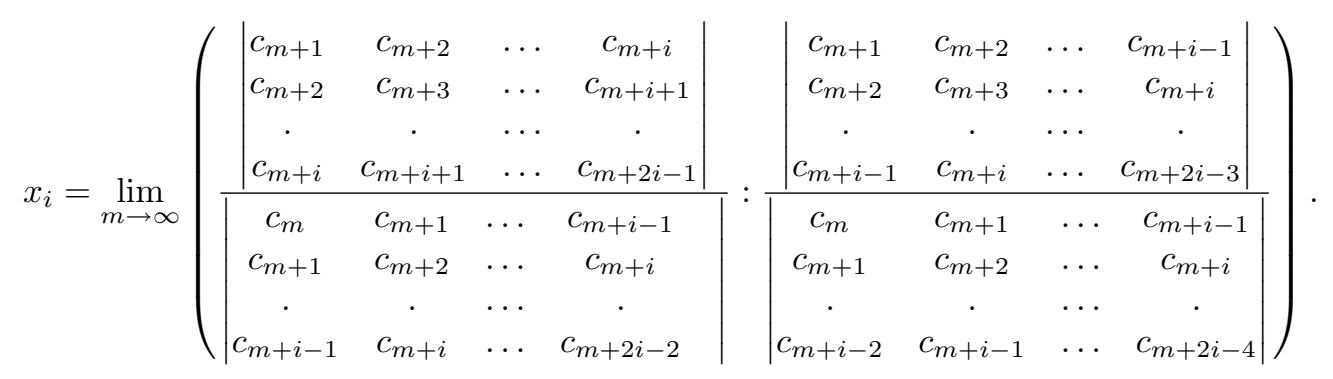

Obviously, only real roots of algebraic equation (1.1) can be found with the use of Aitken formulas. The way to find the largest in magnitude real root of algebraic equation (1.1) described by formula (1.4) was proposed by D.Bernoulli. We suggest to use the $r / \varphi$-algorithm to find complex roots of algebraic equation (1.1).

\section{Representation of zeros of a polynomial}

Let us write Aitken formulas (1.4)-(1.7) in expanded form. We obtain relations that consist of ratios of determinants of Toeplitz matrices. The diagonal elements of determinants are the coefficients of the equation (1.1).

Using recurrent formula (1.3), formula (1.4) can be represented in the form

$$
x_{1}=-\frac{\left|\begin{array}{ccccc}
-\alpha_{1} & -\alpha_{2} & -\alpha_{3} & -\alpha_{4} & \ldots \\
-1 & -\alpha_{1} & -\alpha_{2} & -\alpha_{3} & \ldots \\
0 & -1 & -\alpha_{1} & -\alpha_{2} & \ldots \\
0 & 0 & -1 & -\alpha_{1} & \ldots \\
\cdot & . & . & . & \ldots
\end{array}\right|}{\left|\begin{array}{cccc}
-\alpha_{1} & -\alpha_{2} & -\alpha_{3} & \ldots \\
-1 & -\alpha_{1} & -\alpha_{2} & \ldots \\
0 & -1 & -\alpha_{1} & \ldots \\
. & . & . & \ldots
\end{array}\right|}: \frac{\left|\begin{array}{ccccc}
-1 & -\alpha_{1} & -\alpha_{2} & -\alpha_{3} & \ldots \\
0 & -1 & -\alpha_{1} & -\alpha_{2} & \ldots \\
0 & 0 & -1 & -\alpha_{1} & \ldots \\
0 & 0 & 0 & -1 & \ldots \\
. & . & . & . & \ldots
\end{array}\right|}{\left|\begin{array}{cccc}
-1 & -\alpha_{1} & -\alpha_{2} & \ldots \\
0 & -1 & -\alpha_{1} & \ldots \\
0 & 0 & -1 & \ldots \\
. & . & . & \ldots
\end{array}\right|}
$$

Upon substituting $c_{m}$ into the Aitken formulas, other roots of equation (1.1) are

$$
x_{2}=-\frac{\left|\begin{array}{ccccc}
-\alpha_{2} & -\alpha_{3} & -\alpha_{4} & -\alpha_{5} & \ldots \\
-\alpha_{1} & -\alpha_{2} & -\alpha_{3} & -\alpha_{4} & \ldots \\
-1 & -\alpha_{1} & -\alpha_{2} & -\alpha_{3} & \ldots \\
0 & -1 & -\alpha_{1} & -\alpha_{2} & \ldots \\
. & . & . & . & \ldots
\end{array}\right|}{\left|\begin{array}{cccc}
-\alpha_{2} & -\alpha_{3} & -\alpha_{4} & \ldots \\
-\alpha_{1} & -\alpha_{2} & -\alpha_{3} & \ldots \\
-1 & -\alpha_{1} & -\alpha_{2} & \ldots \\
. & . & . & \ldots
\end{array}\right|}: \frac{\left|\begin{array}{ccccc}
-\alpha_{1} & -\alpha_{2} & -\alpha_{3} & -\alpha_{4} & \ldots \\
-1 & -\alpha_{1} & -\alpha_{2} & -\alpha_{3} & \ldots \\
0 & -1 & -\alpha_{1} & -\alpha_{2} & \ldots \\
0 & 0 & -1 & -\alpha_{1} & \ldots \\
0 & . & . & . & \ldots
\end{array}\right|}{\left|\begin{array}{cccc}
-\alpha_{1} & -\alpha_{2} & -\alpha_{3} & \ldots \\
-1 & -\alpha_{1} & -\alpha_{2} & \ldots \\
0 & -1 & -\alpha_{1} & \ldots \\
. & . & . & \ldots
\end{array}\right|},
$$




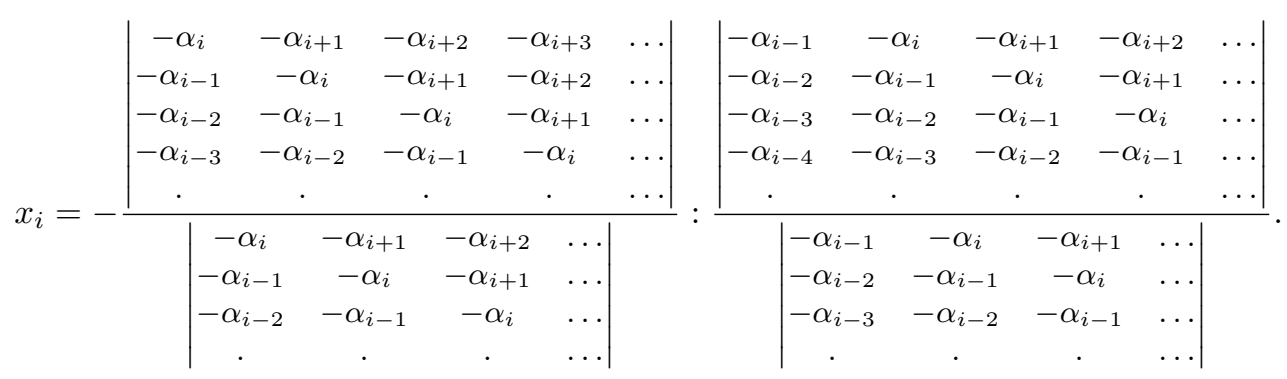

Ratios of determinants (2.1)-(2.3) define the roots of algebraic equation (1.1) in terms of its coefficients. These ratios are referred to as the $N_{i}^{(n)}$ functions. One should note that functions $N_{i}^{(n)}$ for algebraic equations with $n>4$ are written in a similar way to functions for algebraic equations with $n=2,3$ and 4 .

If all roots of equation of $n$-th degree are real then the values of these roots can be found by sequential calculating determinants given in (2.1)-(2.3). Functions $N_{i}^{(n)}$ determined by expressions (2.1)-(2.3) are also referred to as continuous fractions of Nieporte.

\section{The algorithm of summation of divergent continued fractions}

Generalized continuous fractions were proposed [13]. They are defined by the ratio of the determinants of the general form:

$$
w=\frac{v\left|\begin{array}{ccccc}
a_{11} & a_{12} & \ldots & a_{1 n} & \ldots \\
a_{21} & a_{22} & \ldots & a_{2 n} & \ldots \\
. & . & . & . & \ldots \\
a_{n 1} & a_{n 2} & \ldots & a_{n m} & \ldots \\
\ldots & \ldots & \ldots & \ldots & \ldots
\end{array}\right|}{\left|\begin{array}{cccc}
a_{22} & \ldots & a_{2 n} & \ldots \\
. & . & . & \ldots \\
a_{n 2} & \ldots & a_{n m} & \ldots \\
\ldots & \ldots & \ldots & \ldots
\end{array}\right|}
$$

All known classes of continued fractions are special cases of continuous fractions (3.1). For example, branching continuous fractions (3.2), or Skorobogatko continuous fractions [14], are represented by the ratio of determinants with the typical step structure:

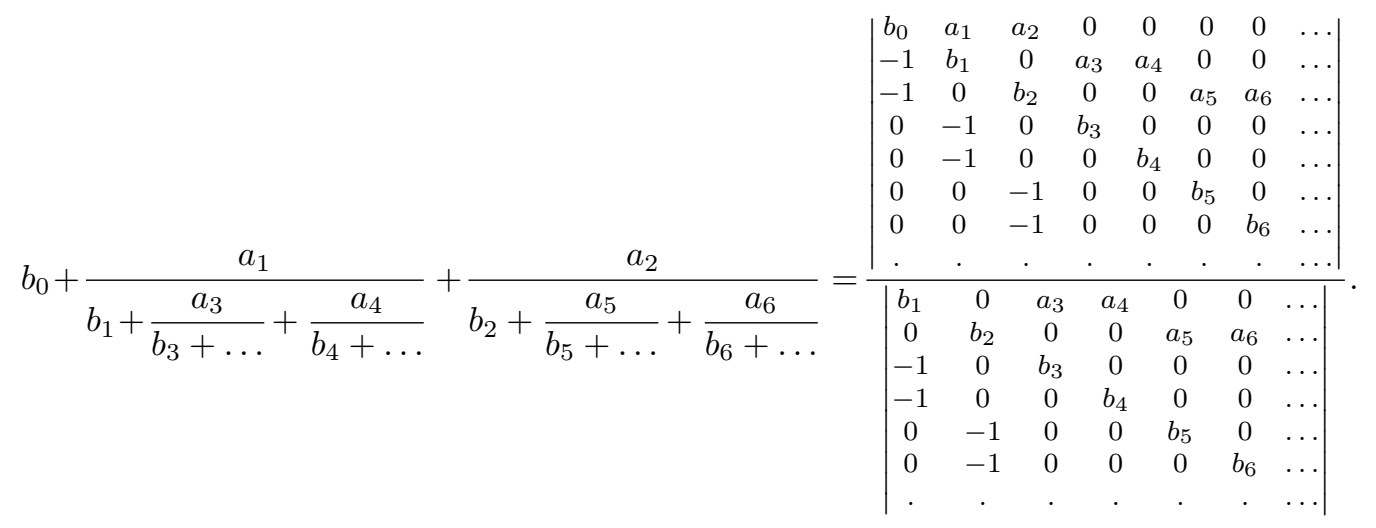


Ordinary continued fractions can be represented by the ratio of determinants of three-diagonal matrices:

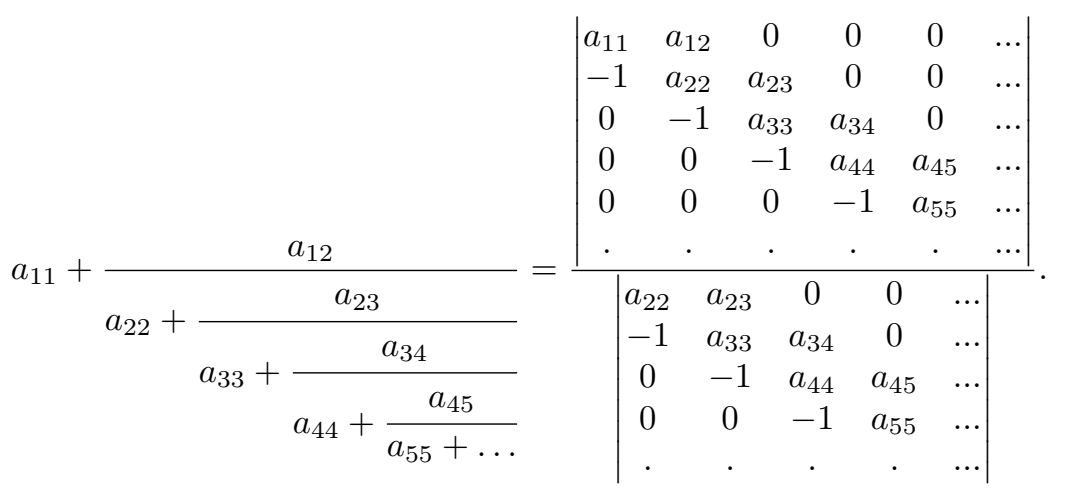

Let us consider the algorithm of determining the values of divergent continued fractions. The algorithm of determining roots of a polynomial is constructed in a similar way. Unconventional definition of convergence of continued fractions was proposed [15]. To find the value of continued fractions we use the $r / \varphi$-algorithm:

Generally continued fraction converges to a complex number $z=r_{0} e^{i \varphi_{0}}$, if there are limits:

$$
\begin{gathered}
\lim _{n \rightarrow \infty} \sqrt[n]{\prod_{l=1}^{n}\left|P_{l} / Q_{l}\right|}=r_{0}, \\
\pi \lim _{n \rightarrow \infty} \frac{k_{n}}{n},=\left|\varphi_{0}\right|,
\end{gathered}
$$

where $P_{l} / Q_{l}$ is the value of l-th appropriate fraction out of $n$ appropriate fractions, $k_{n}$ is the number of negative appropriate fractions out of $n$ fractions

This method goes beyond the traditional methods of summation because it allows one to find a complex number that is represented by this continued fraction from the sequence of real suitable fractions. If appropriate fractions in a continued fraction with real elements change signs any number of times then the continued fraction converges to a complex number.In other words, the behavior of suitable fractions defines the complex number $e^{i}$. Parameters of a complex number $z=r_{0} e^{i \varphi_{0}}$, where $r_{0}$ is the absolute value and $\varphi_{0}$ is the argument, can be determined with the use of $r / \varphi$-algorithm (formulas (3.3) and (3.4)). In the case of continued fractions, converging in the classical meaning, the argument $\varphi_{0}$ takes either the value 0 or $\pi$. If $\varphi_{0}=0$ then the value of convergent continued fraction takes the value $r_{0}$. If $\varphi_{0}=\pi$ then the value of convergent continued fraction takes the value $z=r_{0} e^{i \pi}=-r_{0}$. The scheme of $r / \varphi$-algorithm can be clarified as follows. Let us consider the decomposition of $e^{i \varphi}$ in terms of the continued fraction. From the Euler formula

$$
\cos \varphi=\frac{e^{i \varphi}+e^{-i \varphi}}{2}
$$

continued fractions can be written in the following form:

$$
\begin{gathered}
e^{i \varphi}=2 \cos \varphi-\frac{1}{e^{i \varphi}}, \\
e^{i \varphi}=2 \cos \varphi-\frac{1}{2 \cos \varphi}-\frac{1}{e^{i \varphi}},
\end{gathered}
$$




$$
e^{i \varphi}=2 \cos \varphi-\frac{1}{2 \cos \varphi}-\frac{1}{2 \cos \varphi}-\ldots-\frac{1}{2 \cos \varphi-\frac{1}{e^{i \varphi}}} .
$$

Let us write rational approximants of continued fractions (3.5):

$$
\begin{aligned}
& \frac{P_{1}}{Q_{1}}=2 \cos \varphi=\frac{\sin 2 \varphi}{\sin \varphi}, \\
& \frac{P_{2}}{Q_{2}}=2 \cos \varphi-\frac{1}{2 \cos \varphi}=\frac{\sin 3 \varphi}{\sin 2 \varphi}, \\
& \ldots \ldots \ldots \ldots \ldots \ldots \\
& \frac{P_{n}}{Q_{n}}=2 \cos \varphi-\frac{1}{2 \cos \varphi}-\frac{1}{2 \cos \varphi}-\frac{1}{2 \cos \varphi}-\ldots-\frac{1}{2 \cos \varphi}=\frac{\sin (n+1) \varphi}{\sin n \varphi}
\end{aligned}
$$

Let us depict graphically multiple values of the first suitable fractions of continued frac$\operatorname{tion}(3.5)$.

$$
\begin{aligned}
& \frac{P_{1}}{Q_{1}}=\frac{\sin 2 \varphi}{\sin \varphi}, \\
& \frac{P_{2}}{Q_{2}}=\frac{\sin 3 \varphi}{\sin 2 \varphi},
\end{aligned}
$$

Obviously, angle $(n+1) \varphi$ becomes greater than $\pi$ with increasing $n$ :

$$
\frac{P_{n}}{Q_{n}}=\frac{\sin (n+1) \varphi}{\sin n \varphi}
$$

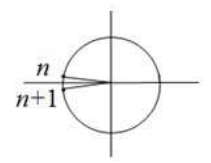

$$
\frac{P_{n}}{Q_{n}}<0 .
$$

At this moment the appropriate fraction $P_{n} / Q_{n}$ takes a negative value and this allows one to approximately determine the argument of a complex number $e^{i \varphi}$ which is presented by continued fraction (3.5). Observing the values of appropriate fractions (3.5), we can write the formula for the argument $\varphi_{0}$ of the complex number $e^{i \varphi}$ :

$$
\varphi_{0}=\frac{\pi k_{n}+\tilde{\varphi}}{n},
$$

where $k_{n}$ is the number of appropriate fractions that have a negative value out of the total number $n$ of fractions (3.5), $\tilde{\varphi}$ is some angle and $\tilde{\varphi}<\varphi_{0}$. 
If $n \rightarrow \infty$ then formula (3.7) takes the form

$$
\varphi_{0}=\pi \lim _{n \rightarrow \infty} \frac{k_{n}}{n} .
$$

However, the above procedure allows one to determine not the value of argument of the complex number $e^{i \varphi_{0}}$ but the absolute value of this argument. The sign of the argument of a complex number $e^{i \varphi_{0}}$ is determined by the dynamics of distribution of values of appropriate fractions (3.5) on the "period". These rules of determining the sign were installed after calibration on the test continued fractions with complex values.

Fig. 1 and 1a show the distribution of values of appropriate fractions $P_{n} / Q_{n}$ of expansions $e^{i 0,2}$ and $e^{-i 0,2}$.

$$
\begin{gathered}
2 \cos 0.2-\frac{1}{2 \cos 0.2}-\frac{1}{2 \cos 0.2}-\cdots-\frac{1}{2 \cos 0.2}=\frac{\sin (n+1) 0.2}{\sin n 0.2} . \\
\frac{1}{2 \cos 0.2}-\frac{1}{2 \cos 0.2}-\cdots-\frac{1}{2 \cos 0.2}=\frac{\sin n 0.2}{\sin (n+1) 0.2} .
\end{gathered}
$$
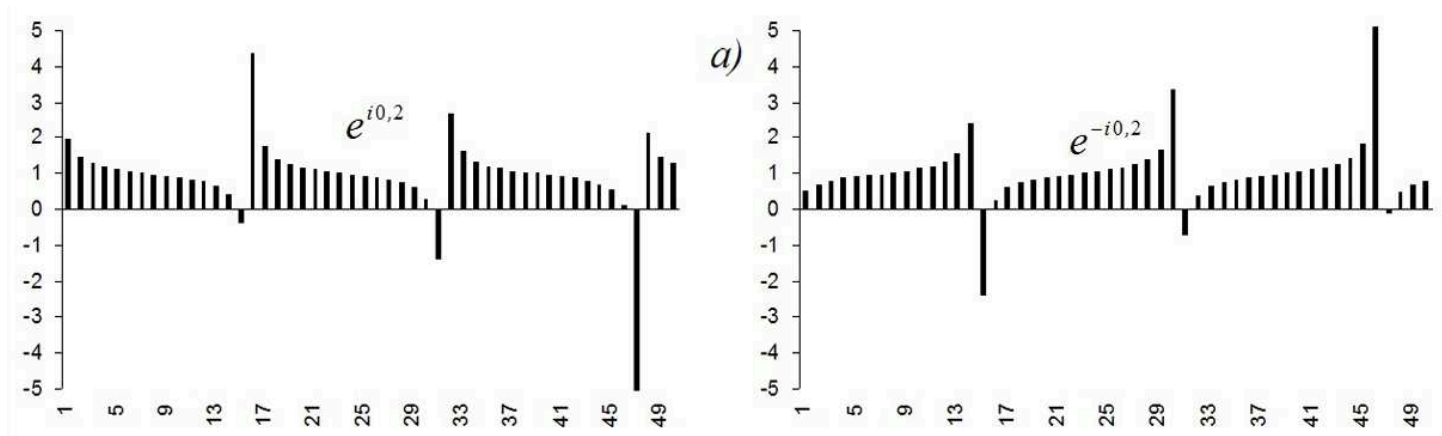

Fig. 1. The distribution of values of appropriate continued fractions (3.8) and (3.9)

Formula (3.3) is assigned in a similar way.

\section{The algorithm of determination of roots of a polynomial}

Relations (2.1)-(2.3) are continued fractions with special structure. This allows one to introduce the concept of an appropriate fraction and it simplifies the description of the method of solution of algebraic equations with the use of functions $N_{l}^{(n)}$ and $r / \varphi$-algorithm.

To find a complex roots of equation (1.1) that are also determined by formulas (2.1)-(2.3) it is necessary to use the $r / \varphi$-algorithm. Modulus $r_{l}$ and the absolute value of argument $\varphi_{l}$ of a complex number $x_{l}=r_{l} e^{i \varphi_{l}}$ are determined from the formulas:

$$
\begin{gathered}
r_{l}=\lim _{m \rightarrow \infty} \sqrt[m]{\prod_{m=1}^{m}\left|\bar{x}_{l}^{(m)}\right|}, l=1,2, \ldots, n . \\
\left|\varphi_{l}\right|=\pi \lim _{m \rightarrow \infty} \frac{k_{l}^{(m)}}{m},
\end{gathered}
$$

where $\bar{x}_{l}^{(m)} i s m$-th appropriate fraction of expression $(2.3)$ and $k_{l}^{(m)}$ is a number of negative appropriate fractions for the $l$-th root out of $m$ suitable fractions. 
For example, appropriate fractions for $x_{2}$ are as follows:

$$
\begin{gathered}
\bar{x}_{2}^{(1)}=-\frac{\left|-\alpha_{2}\right|}{1}: \frac{\left|-\alpha_{1}\right|}{1}, \bar{x}_{2}^{(2)}=\frac{\left|\begin{array}{ll}
-\alpha_{2} & -\alpha_{3} \\
-\alpha_{1} & -\alpha_{2}
\end{array}\right|}{\left|-\alpha_{2}\right|}: \frac{\left|\begin{array}{ll}
-\alpha_{1} & -\alpha_{2} \\
-1 & -\alpha_{1}
\end{array}\right|}{\left|-\alpha_{1}\right|}, \\
\bar{x}_{2}^{(3)}=\frac{\left|\begin{array}{lll}
-\alpha_{2} & -\alpha_{3} & -\alpha_{4} \\
-\alpha_{1} & -\alpha_{2} & -\alpha_{3} \\
-1 & -\alpha_{1} & -\alpha_{2}
\end{array}\right|}{\left|\begin{array}{ll}
-\alpha_{2} & -\alpha_{3} \\
-\alpha_{1} & -\alpha_{2}
\end{array}\right|}: \frac{\left|\begin{array}{ccc}
-\alpha_{1} & -\alpha_{2} & -\alpha_{3} \\
-1 & -\alpha_{1} & -\alpha_{2} \\
0 & -1 & -\alpha_{1}
\end{array}\right|}{\left|\begin{array}{cc}
-\alpha_{1} & -\alpha_{2} \\
-1 & -\alpha_{1}
\end{array}\right|}, \ldots .
\end{gathered}
$$

Calculation of appropriate fractions with the use of formula (2.3) is very difficult due to the large dimensions of the determinants which are included in this formula. However, it is easy to see that the determinants presented in formula (2.3) have special structure. They are determinants of Toeplitz matrices in which the elements on the diagonals parallel to the main diagonal are the same. To implement formulas (2.3) one can use the quotient-difference (QD) algorithm proposed by Rutishauser [16].

\section{An example of solving algebraic equatins using the $r / \varphi$-algorithm and the results of testing}

Tables 1-5 present the results of calculations of the roots of the equation

$$
x^{12}-x^{11}-x^{10}-x^{9}-x^{8}-x^{7}-x^{6}-x^{5}-x^{4}-x^{3}-x^{2}-x-1=0
$$

with the use of continued fractions (2.3) and $r / \varphi$-algorithm (4.1) and (4.2).

The limit of the ratio of determinants which is included in formula (5.2) coincides with the largest magnitude root of equation (5.1):

$$
x_{1}=\frac{\left|\begin{array}{ccccccc}
1 & 1 & 1 & \ldots & 1 & 0 & \ldots \\
-1 & 1 & 1 & \ldots & 1 & 1 & \ldots \\
0 & -1 & 1 & \ldots & 1 & 1 & \ldots \\
. & . & . & \ldots & . & . & \ldots \\
0 & 0 & 0 & \ldots & 1 & 1 & \ldots \\
0 & 0 & 0 & \ldots & -1 & 1 & \ldots \\
. & . & . & \ldots & . & . & \ldots
\end{array}\right|}{\left|\begin{array}{cccccc}
1 & 1 & \ldots & 1 & 1 & \ldots \\
-1 & 1 & \ldots & 1 & 1 & \ldots \\
. & . & \ldots & . & . & \ldots \\
0 & 0 & \ldots & 1 & 1 & \ldots \\
0 & 0 & \ldots & -1 & 1 & \ldots \\
. & . & \ldots & . & . & \ldots
\end{array}\right|}=1.99975500937 \ldots
$$

The results of calculation of real root $x_{1}$ of equation (5.1) are given in Tab. 1.

The data in Tab. 1 show high rate of convergence of continued fraction (5.2) which represents the real root of equation (5.1). 
Table 1. The results of calculation real root $x_{1}$

$x_{1}=1.99975500937 \ldots$

\begin{tabular}{|c|c|c|c|c|c|}
\hline $\begin{array}{c}\text { Number } \\
\text { of fraction } \\
\text { components }\end{array}$ & $\begin{array}{c}\text { Value of the } \\
\text { the appropriate } \\
\text { fraction }\end{array}$ & $\begin{array}{c}\text { Error, } \\
r_{0}-r_{i}\end{array}$ & $\begin{array}{c}\text { Number } \\
\text { of fraction } \\
\text { components }\end{array}$ & $\begin{array}{c}\text { Value of the } \\
\text { the appropriate } \\
\text { fraction }\end{array}$ & $\begin{array}{c}\text { Error, } \\
r_{0}-r_{i}\end{array}$ \\
\hline 0 & 1.000000000000 & 0.9997555009370 & 22 & 1.999755531109 & -0.000000030172 \\
\hline 1 & 2.000000000000 & -0.000244499063 & 23 & 1.999755501222 & -0.000000000285 \\
\hline 12 & 1.999511718750 & -0.000243782187 & 25 & 1.999755501135 & -0.000000000198 \\
\hline 13 & 1.999755799756 & $-0,000000298819$ & 26 & 1.999755501098 & -0.000000000161 \\
\hline 14 & 1.999755769935 & -0.000268998000 & 27 & 1.999755501066 & -0.000000000129 \\
\hline 15 & 1.999755740107 & -0.000000239170 & 28 & 1.999755501036 & -0.000000000099 \\
\hline 16 & 1.999755710272 & -0.000000209335 & 29 & 1.999755501011 & -0.000000000074 \\
\hline 17 & 1.999755680430 & -0.000000179493 & 30 & 1.999755500989 & -0.000000000052 \\
\hline 18 & 1.999755650580 & -0.000000149643 & 31 & 1.999755500970 & -0.000000000033 \\
\hline 19 & 1.999755620723 & -0.000000119786 & 32 & 1.999755500956 & -0.000000000019 \\
\hline 20 & 1.999755590859 & -0.000000089922 & 33 & 1.999755500945 & -0.000000000008 \\
\hline 21 & 1.999755560988 & -0.000000060051 & 34 & 1.999755500938 & -0.000000000001 \\
\hline
\end{tabular}

Let us write continued fraction for the roots $x_{2}$ and $x_{3}$ of equation (5.1):

$$
\begin{aligned}
& x_{2}=-\frac{\left|\begin{array}{cccccccc}
1 & 1 & 1 & 1 & \ldots & 0 & 0 & \ldots \\
1 & 1 & 1 & 1 & \ldots & 1 & 0 & \ldots \\
-1 & 1 & 1 & 1 & \ldots & 1 & 1 & \ldots \\
0 & -1 & 1 & 1 & \ldots & 1 & 1 & \ldots \\
. & . & . & . & \ldots & . & . & \ldots \\
0 & 0 & 0 & 0 & \ldots & 1 & 1 & \ldots \\
0 & 0 & 0 & 0 & \ldots & 1 & 1 & \ldots \\
. & . & . & . & \ldots & . & . & \ldots
\end{array}\right|}{\left|\begin{array}{ccccccccccc}
1 & 1 & 1 & \ldots & 1 & 0 & \ldots \\
1 & 1 & 1 & \ldots & 1 & 1 & \ldots \\
-1 & 1 & 1 & \ldots & 1 & 1 & \ldots \\
. & . & . & \ldots & . & . & \ldots \\
0 & 0 & 0 & \ldots & 1 & 1 & \ldots \\
0 & 0 & 0 & \ldots & 1 & 1 & \ldots \\
. & . & . & \ldots & . & . & \ldots
\end{array}\right|}: \frac{\left|\begin{array}{cccccccc}
1 & 1 & 1 & 1 & \ldots & 1 & 0 & \ldots \\
-1 & 1 & 1 & 1 & \ldots & 1 & 1 & \ldots \\
0 & -1 & 1 & 1 & \ldots & 1 & 1 & \ldots \\
0 & 0 & -1 & 1 & \ldots & 1 & 1 & \ldots \\
. & . & . & . & \ldots & . & . & \ldots \\
0 & 0 & 0 & 0 & \ldots & 1 & 1 & \ldots \\
0 & 0 & 0 & 0 & \ldots & -1 & 1 & \ldots \\
. & . & . & . & \ldots & . & . & \ldots
\end{array}\right|}{\left|\begin{array}{ccccccc}
1 & 1 & 1 & \ldots & 1 & 1 & \ldots \\
-1 & 1 & 1 & \ldots & 1 & 1 & \ldots \\
0 & -1 & 1 & \ldots & 1 & 1 & \ldots \\
. & . & . & \ldots & . & . & \ldots \\
0 & 0 & 0 & \ldots & 1 & 1 & \ldots \\
0 & 0 & 0 & \ldots & -1 & 1 & \ldots \\
. & . & . & \ldots & . & . & \ldots
\end{array}\right|},
\end{aligned}
$$

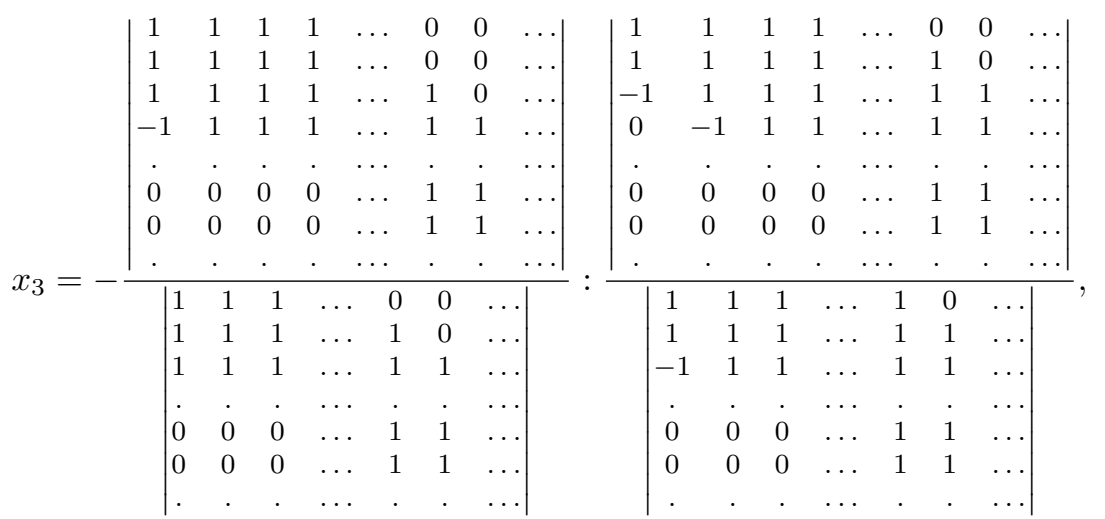

Fig. 2 and Fig. 3 show the graphs of the values of appropriate continued fractions which are roots of algebraic equation (5.1). The graphs $2 a$ and $3 m$ shows that $x_{1}$ и $x_{12}$ are real roots. The graphs also show that equation (5.1) has five pairs of complex conjugate roots. "Periodicity" of the location of suitable complex roots can be clearly seen at the right half of the graphs presented in Fig. 2 and Fig. 3. 
Tab. 2 and 3 show the results of calculating of the first pair of complex conjugate roots of equation (5.1) with the use of $r / \varphi$-algorithm (formulas (4.1) and (4.2)).

Table 2. The results of calculations of the complex root $x_{2}$

$x_{2}=0.979690425957 e^{i 0.558419353144} \ldots$

\begin{tabular}{|c|c|c|c|c|c|}
\hline $\begin{array}{c}\text { Number } \\
\text { of fraction } \\
\text { components }\end{array}$ & $\begin{array}{c}\text { Value of } \\
\text { the appropriate } \\
\text { fraction }\end{array}$ & $\begin{array}{c}\text { Absolute } \\
\text { value, } r_{i}\end{array}$ & $\begin{array}{c}\text { Error of } \\
\text { absolute value, } \\
r_{0}-r_{i}\end{array}$ & $\begin{array}{c}\text { Value of } \\
\text { the argument, } \\
\varphi_{i}\end{array}$ & $\begin{array}{c}\text { Error of } \\
\text { the argument, } \\
\varphi_{0}-\varphi_{i}\end{array}$ \\
\hline 32 & 1.501296168008 & 0.864581149632 & 0.115109276325 & 0.598398600684 & -0.039979247540 \\
\hline 64 & 1.206754380797 & 0.929375999911 & 0.050314426046 & 0.592753330866 & -0.034333977722 \\
\hline 128 & 0.790280743715 & 0.958447588838 & 0.021242837119 & 0.563875604490 & -0.005456251346 \\
\hline 256 & 1.182647495564 & 0.970361670301 & 0.009328755656 & 0.564204394930 & -0.005785041786 \\
\hline 512 & 0.040108503235 & 0.967761878002 & 0.011928547955 & 0.558087317704 & 0.000332035440 \\
\hline 1024 & -0.006146637028 & 0.971932508313 & 0.007757917644 & 0.561330967719 & -0.002911614575 \\
\hline 2048 & -0.111326789935 & 0.977170978893 & 0.002519447064 & 0.559841989815 & -0.001422636671 \\
\hline 4096 & -0.395348926941 & 0.978685754271 & 0.001004671686 & 0.559103515094 & -0.000684161950 \\
\hline
\end{tabular}

Table 3. The results of calculations of the complex root $x_{3}$

$x_{3}=0.979690425957 e^{-i 0.558419353144} \ldots$

\begin{tabular}{|c|c|c|c|c|c|}
\hline $\begin{array}{c}\text { Number } \\
\text { of fraction } \\
\text { components }\end{array}$ & $\begin{array}{c}\text { Value of } \\
\text { the appropriate } \\
\text { fraction }\end{array}$ & $\begin{array}{c}\text { Absolute } \\
\text { value, } r_{i}\end{array}$ & $\begin{array}{c}\text { Error of } \\
\text { absolute value, } \\
r_{0}-r_{i}\end{array}$ & $\begin{array}{c}\text { Value of } \\
\text { the argument, } \\
\varphi_{i}\end{array}$ & $\begin{array}{c}\text { Error of } \\
\text { the argument, } \\
\varphi_{0}-\varphi_{i}\end{array}$ \\
\hline 32 & 2.664690114532 & 0.602084440858 & 0.377605985099 & -0.698131700798 & 0.139712347654 \\
\hline 64 & 1.691424133396 & 0.871677635662 & 0.108012790295 & -0.612993688505 & 0.054574335361 \\
\hline 128 & 1.376117926277 & 0.953841250747 & 0.025849175210 & -0.568478670650 & 0.010059317506 \\
\hline 256 & 0.814122533036 & 0.966453199595 & 0.013237226362 & -0.566295671463 & 0.007876318319 \\
\hline 512 & 23.92984168573 & 0.980954443190 & -0.001264017233 & -0.558933662295 & 0.000514309151 \\
\hline 1024 & $-156,1493425219$ & 0.982185980387 & -0.002495554430 & -0.561783301691 & 0.003363948547 \\
\hline 2048 & -8.621404886212 & 0.979563790294 & 0.000126635663 & -0.560056764418 & 0.001637411274 \\
\hline 4096 & -2.427711991375 & 0.979374005615 & 0.000316420342 & -0.559208120268 & 0.000788767124 \\
\hline
\end{tabular}

Tab. 4 shows the results of calculations of complex roots of equation (5.1) by formulas (4.1) and (4.2) with the use of 4096 appropriate fractions.

Table 5 shows the results of calculation of the second real root of equation (5.1).

Next we present the results of testing of $r / \varphi$-algorithm. The following equation with random coefficients is considered:

$$
x^{10}+a_{1} x^{9}+a_{2} x^{8}+\cdots++a_{9} x+a_{10}=0
$$

where $a_{1}, a_{2}, \ldots, a_{10} \in[-1000000,1000000]$.

To calculate the appropriate fractions QD-algorithm with negative indices is used. It is required to find out whether a given accuracy is achieved in the determination of real and complex roots of equations (5.5) with the use of $r / \varphi$-algorithm (formulas (4.1) and (4.2)). The performed calculations confirm the validity of the $r / \varphi$-algorithm for finding zeros of a polynomial. We applied the $r / \varphi$-algorithm to 10000 algebraic equations of tenth degree with random coefficients. Relative error of all roots calculated is no more than 0.001 . Convergence to the exact solution takes place for all equations and a given accuracy is achieved. The number of appropriate fractions needed to achieve the required accuracy depends on specific equation. 
Table 4. The results of the calculation of complex roots of equation (5.1)

\begin{tabular}{|c|c|c|c|c|}
\hline $\begin{array}{c}\text { Number } \\
\text { of fraction } \\
\text { components }\end{array}$ & $\begin{array}{c}\text { Absolute } \\
\text { value, } \\
r_{i}\end{array}$ & $\begin{array}{c}\text { Error of } \\
\text { absolute value, } \\
r_{0}-r_{i}\end{array}$ & $\begin{array}{c}\text { Value of } \\
\text { the argument, } \\
\varphi_{i}\end{array}$ & $\begin{array}{c}\text { Error of } \\
\text { the argument, } \\
\varphi_{0}-\varphi_{i}\end{array}$ \\
\hline$x_{2}$ & 0.978685754271 & 0.001004671686 & 0.559103515094 & -0.000684161950 \\
\hline$x_{3}$ & 0.979374005615 & 0.000316420342 & -0.559208120268 & 0.000788767124 \\
\hline$x_{4}$ & 0.952951183889 & 0,000484993278 & 1.089229858718 & $-0,000627378152$ \\
\hline$x_{5}$ & 0.952945508991 & 0.000490668176 & -1.089354429647 & 0.000751949081 \\
\hline$x_{6}$ & 0.933794454170 & 0.001277830104 & 1.606982618197 & -0.000290761923 \\
\hline$x_{7}$ & 0.935996065142 & -0.000923780868 & -1.607090502793 & 0.000398646519 \\
\hline$x_{8}$ & 0,923441518037 & -0.000096550663 & 2,120751183298 & -0.000751101152 \\
\hline$x_{9}$ & 0.923354809862 & -0.000009842488 & -2.120830231779 & 0.000830149633 \\
\hline$x_{10}$ & 0.916414755115 & 0.000396701291 & 2.631251204724 & -0.000039654711 \\
\hline$x_{11}$ & 0.917193803082 & -0.000382346676 & -2.631291206716 & 0.000079656703 \\
\hline
\end{tabular}

Table 5. The results of calculation of real root $x_{12}$

$x_{12}=-0.914710602925 \ldots$

\begin{tabular}{|c|c|c|c|c|c|}
\hline $\begin{array}{c}\text { Number } \\
\text { of fractions } \\
i\end{array}$ & $\begin{array}{c}\text { Value of } \\
\text { the appropriate } \\
\text { fraction }\end{array}$ & $\begin{array}{c}\text { Error, } \\
r_{0}-r_{i}\end{array}$ & $\begin{array}{c}\text { Number } \\
\text { of fractions } \\
i\end{array}$ & $\begin{array}{c}\text { Value of } \\
\text { the appropriate } \\
\text { fraction }\end{array}$ & $\begin{array}{c}\text { Error, } \\
r_{0}-r_{i}\end{array}$ \\
\hline 120 & -0.166666666667 & -0.748043936258 & 794 & -0.915108995829 & 0.000398392904 \\
\hline 121 & -0.369230769231 & -0.545479833694 & 1151 & -0.915064419461 & 0.000353816536 \\
\hline 122 & -0.619047619048 & -0.295662983877 & 1428 & -0.914696626243 & -0.000013976682 \\
\hline 123 & -0.933333333333 & 0.018622730408 & 2339 & -0.914714604778 & 0.000004001853 \\
\hline 246 & -0.900000540829 & -0.014710062096 & 2973 & -0.914712028919 & 0.000001425994 \\
\hline 283 & -0.911768355829 & -0.002942247096 & 3250 & -0.914711862234 & 0.000001259309 \\
\hline 517 & -0.917558586942 & 0.002847984017 & 3607 & -0.914709718575 & -0.000000884350 \\
\hline 677 & -0.912638347559 & -0.002072255366 & 3884 & -0.914710601151 & -0.000000001774 \\
\hline
\end{tabular}

\section{Conclusion}

Formulas (2.3), (4.1) and (4.2) define the roots of a polynomial of the $n$-th degree in terms of its coefficients. Using these formulas, one can establish various criteria associated with the roots of polynomials of general form. Numerical methods are not capable of solving such problems. The fact that formula (2.3) includes the determinants of infinite order should not cause additional concerns because even the finding of roots of a quadratic equation includes the computation of the ratio of determinants of infinite three-diagonal matrices of dimensions $(n+1)$ and $n$ with $n \rightarrow \infty$. Formula (2.3) includes the ratio of Toeplitz determinants of infinite order and it can be considered as a mnemonic rule of finding the roots of a random algebraic equation of $n$-th order which is transformed into sequence of arithmetic operations. Similarly the formulas for finding the roots of quadratic or cubic equations are also mnemonic rules. Formula (2.3) is referred as function $N_{i}^{(n)}$. Arbitrary algebraic equation is not solvable in radicals, but it is solvable with the use of $r / \varphi$-algorithm (formulas (4.1) and (4.2)) in terms of functions $N_{i}^{(n)}$. The functions are defined by the ratios of determinants of Toeplitz matrices of infinite order (2.3).

Application of the $r / \varphi$-algorithm to functions $N_{i}^{(n)}$, that is to relation $(2.3)$, which contains only real coefficients of an algebraic equation of $n$-th degree allows one to "extract" complex roots of this equation if the complex roots exist. This is paradoxical result that does not fit with 
the classical approach to represent complex numbers in the "explicit" form which contains the imaginary unit. The use of the $r / \varphi$-algorithm allows one to establish the existence of complex roots from the "behavior" of appropriate fractions. The complex roots are determined from "expanding" continued fractions (2.3) with the use of the $r / \varphi$-algorithm. Fig. 4 shows the values

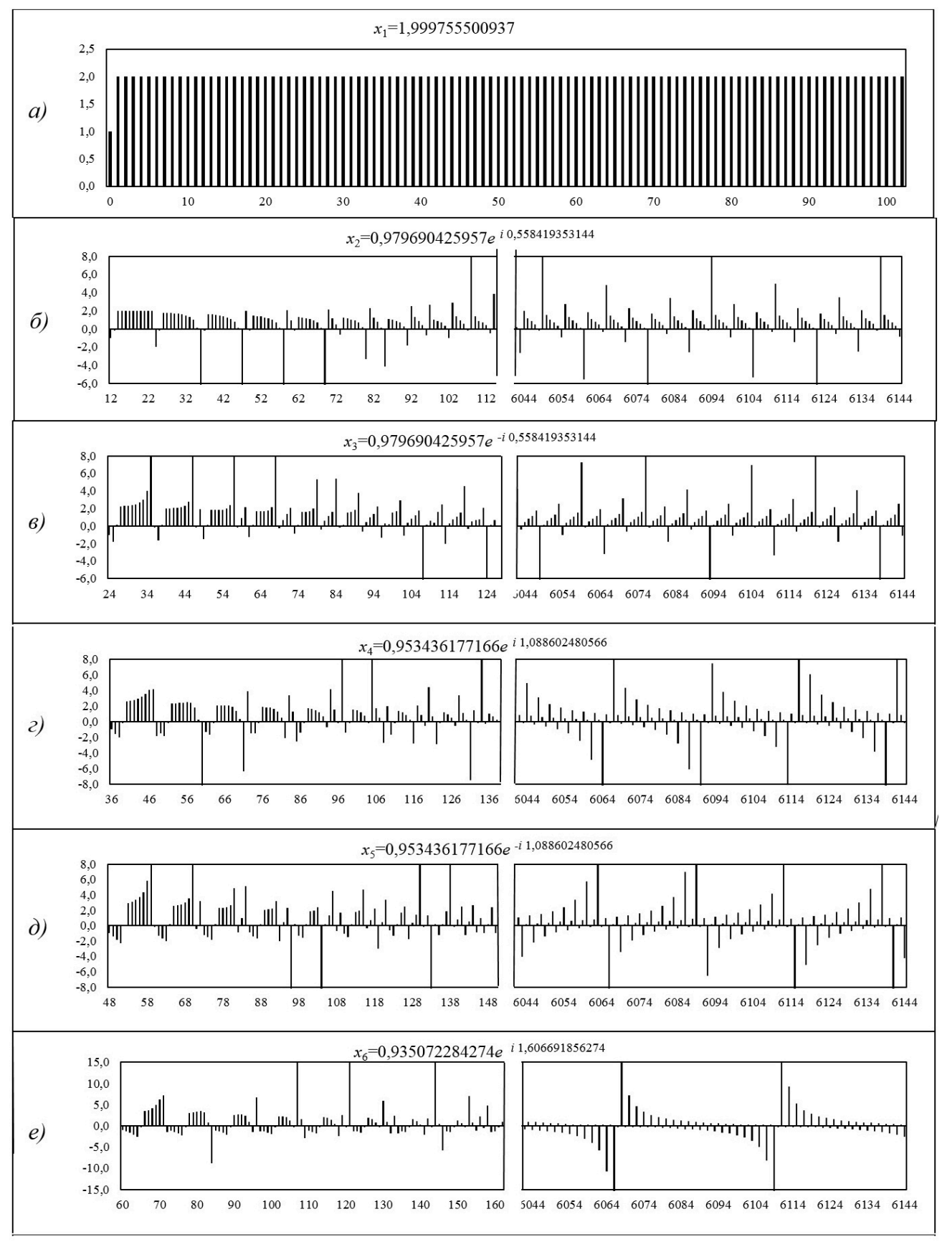

Fig. 2. The distribution of values of appropriate fractions that represent the roots of algebraic equation (5.1) 


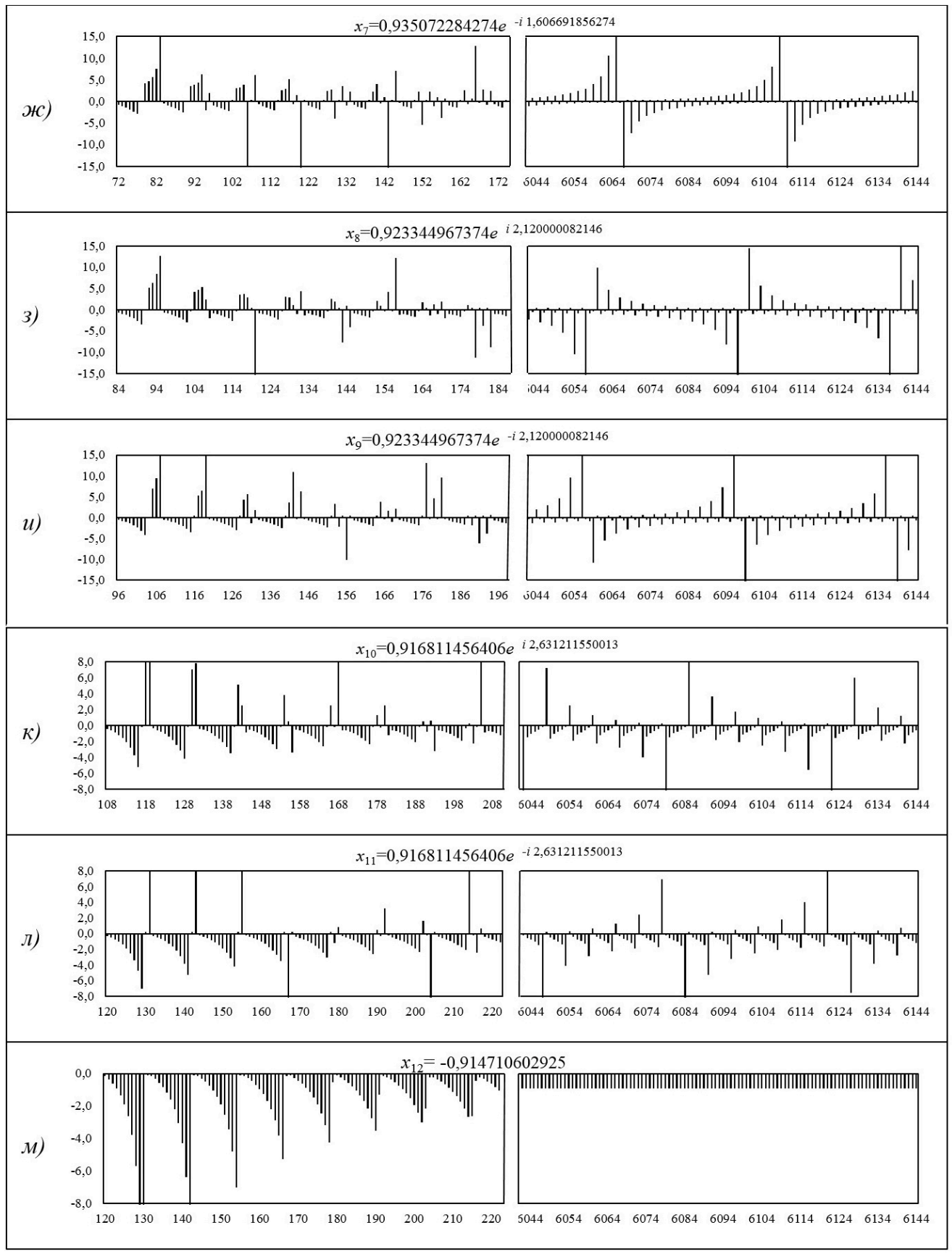

Fig. 3. The distribution of values of appropriate fractions that represent the roots of algebraic equation (5.1)

of roots of equation (5.1) on the complex plane. The $r / \varphi$-algorithm requires a large amount of calculations because complex roots are determined from the analysis of a long series of appropriate continued fractions that have various patterns. The elements of these continued fractions are real. 


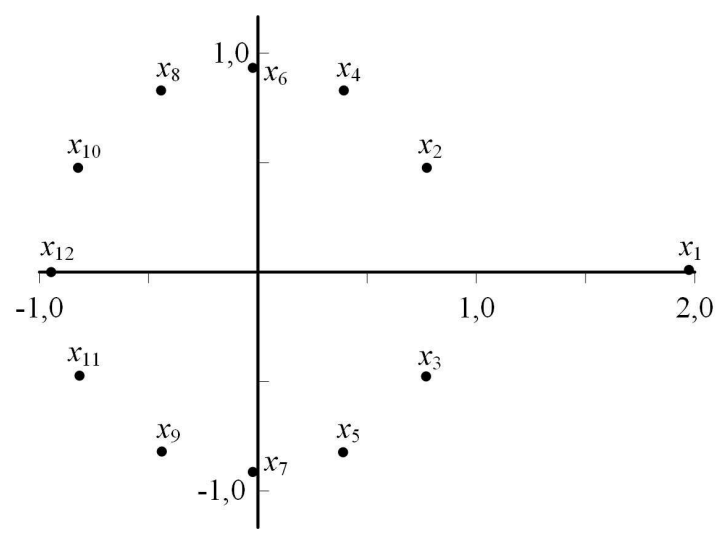

Fig. 4. Locations of roots of equation (5.1) on the complex plane

\section{References}

[1] G.P.Katishev, Solution of algebraic equations of arbitrary degree: theory, methods, algorithms, URRS, Moscow, 2010 (in Russian).

[2] I.F.Korchagin, Algebraic equations, Fizmatkniga, Moscow, 2006 (in Russian).

[3] P.W.Hamming, Numerical methods for scientists and engineers, McGraw-Hill, New York, 1962.

[4] V.I.Shmoylov, R.I.Tuchapsky, Algebraic equations. An infinite system of linear algebraic equations. Bibliographic index, Mercator, Lviv, 2003 (in Russian).

[5] H.J.Mellin, Resolution de l' equation algebrique generale a l'aide de gamma function, C.R. Acad. Sci. Paris, Ser. I Math., 172(1921), 658-661.

[6] E.N.Mikhalkin, On solving general algebraic equations by integrals of elementary functions, Siberian Math. J., 47(2006), no. 2, 301-306

[7] V.I.Shmoylov, Continuous fraction, Divergent continuous fraction, Inst. problems of mechanics and mathematics. NAN of Ukraine, Mercator, Lviv, 2004 (in Russian).

[8] V.I.Shmoylov, V.B.Kovalenko, Some applications of the algorithm of summation of divergent continued fractions, Bulleten Yuzhnogo nauchnogo tsentra RAN, 8(2012),no.4, 3-13 (in Russian).

[9] V.I.Shmoylov, D.E.Savchenko, About the algorithm of summation of divergent continued fractions, Vestnik Voronezhskogo Gos. Universiteta. Series: Fizika. Matematika, (2013), no. 2, 258-276 (in Russian).

[10] V.I.Shmoylov, Divergent systems of linear algebraic equations, Taganrog. tehnolog. institut Yuzhhogo federal. universiteta, Taganrog, 2010 (in Russian).

[11] V.F.Guzik, V.I.Shmoylov, G.A.Kirichenko, Continuous fractions and their application in computational mathematics, Izvestiya YuFedU. Engenernye Nauki, 150(2014), no. 1, 158-174 (in Russian).

[12] A.C.Aitken, On Bernulli's numerical solution of algebraic equations, Edinburg, Proc. Roy. Soc., 1925/26, 289-305. 
[13] V.I.Shmoylov, Continuous fractions and $r / \varphi$-algorithm, TSURE, Taganrog, 2012 (in Russian).

[14] V.A.Skorobogatko, Theory of branched continued fractions and its applications in computational mathematics, Nauka, Moscow, 1983 (in Russian).

[15] V.I.Shmoylov, Periodic continued fractions, Academic Express, Lviv, 1998 (in Russian).

[16] H.Rutishauser, Der Quotienten-Differenzen-Algorithmu, Birkhauser, Basel, 1957.

\section{Решение алгебраических уравнений непрерывными дробями Никипорца}

Владимир И. Шмойлов Геннадий А. Кириченко

Приводятся аналитические выражения, представляющие все корни произвольного алгебраического уравнения $n$-й степени через коэффициенты исходного уравнения. Эти формулы состоят из двух отношений бесконечных определителей Теплица, диагоналъными элементами которъх являются коэфбициенты алгебраического уравнения. Для нахождения комплексных корней дополнительно используется метод суммирования расходящихся непрерывных дробей.

Ключевые слова: алгебраические уравнения, бесконечные определители Теплица, расходящи- еся

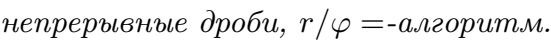

